\title{
Atmospheric Retrieval of Cloudy Venus-Twin Exoplanets in the Context of the LIFE Mission
}

\section{Other Conference Item}

\section{Author(s):}

Konrad, Björn (D); Alei, Eleonora (D); Angerhausen, Daniel; Quanz, Sascha Patrick (D); LIFE Collaboration

Publication date:

2021-09

Permanent link:

https://doi.org/10.3929/ethz-b-000523074

Rights / license:

Creative Commons Attribution 4.0 International

Originally published in:

EPSC Abstracts 15, https://doi.org/10.5194/epsc2021-578 


\title{
Atmospheric Retrieval of Cloudy Venus-Twin Exoplanets in the Context of the LIFE Mission
}

\author{
Björn S. Konrad ${ }^{1}$, Eleonora Alei ${ }^{1}$, Daniel Angerhausen ${ }^{1}$, Sascha P. Quanz ${ }^{1}$, and the LIFE \\ collaboration* \\ ${ }^{1}$ ETH Zurich, Institute for Particle Physics and Astrophysics, Wolfgang Pauli Strasse 27, $\mathrm{CH}-8093$ Zurich, Switzerland \\ (konradb@student.ethz.ch) \\ ${ }^{*}$ A full list of authors appears at the end of the abstract
}

\section{Context}

Current knowledge suggests that temperate terrestrial exoplanets commonly occur in our galaxy (Bryson et al., 2021). One long-term goal of exoplanet research is to characterize the atmospheres of a sizable sample of temperate planets. Such measurements will add to our knowledge of the diversity of worlds in our galaxy, which is a driving force behind this effort, and they will enable the discovery of habitable worlds.

The sensitivity of currently planned space- and ground-based observatories will unlikely allow for the characterization of a large number of temperate exoplanet atmospheres. Concepts for future missions (e.g. NASA's LUVOIR [a] and HabEx [b]), capable of characterizing exoplanet atmospheres in reflected light (optical and near-infrared (NIR) range), have been proposed. Complementary to these efforts, the LIFE mission introduced in Quanz et al. (2018) (see also Angerhausen et al. (2021, this conference) for a general overview) is a proposed space-based nulling interferometer which measures the mid-infrared (MIR) thermal emission of exoplanets.

The MIR regime is particularly powerful, since it provides probes for atmospheric molecules not accessible in the optical or NIR (e.g. the biosignatures $\mathrm{CH}_{4}$ and $\mathrm{N}_{2} \mathrm{O}$ ). Furthermore, MIR emission observations, in contrast to optical and NIR observations, allow us to probe the temperature structure, radius and surface conditions on exoplanets (Quanz et al., 2019; Konrad et al. in prep.). Previous work has focussed on constraining technical requirements for LIFE via retrievals of cloudfree Earth-twin MIR spectra (Konrad et al., 2020; Konrad et al. in prep.). We now generalize these estimates by looking beyond the cloud-free Earth-twin. Specifically we consider Venus (this submission) and Earth's evolution through time (Alei et al. (2021, this conference)).

\section{Methods}

Our approach is based on the Bayesian retrieval of simulated mock observations with LIFE for a Venus-twin exoplanet around a sun-like star located at 10 pc. We generate Venus' emission spectrum using the 1D radiative transfer tool petitRADTRANS (Mollière et al. 2019). The model takes the planetary radius and mass, the pressure-temperature (PT) profile, the atmospheric composition and cloud properties as input. We parametrize Venus' PT profile via a 3rd order polynomial and consider line and collision-induced absorption by $\mathrm{H}_{2} \mathrm{O}, \mathrm{CO}_{2}$, and $\mathrm{CO}$ as well as absorption and 
emission contributions from Venus' opaque $\mathrm{H}_{2} \mathrm{SO}_{4}$ clouds.

With LIFESim(Ottiger et al. in prep.), we simulate the wavelength-dependent SNR expected for observations with LIFE for a Venus-twin. LIFESim considers noise contributions from stellar leakage, local zodiacal dust, and exozodiacal dust.

Our retrieval mechanism is built upon the Multinest algorithm (Feroz et al., 2009). Using the LIFESim noise estimate as uncertainty on the Venus spectrum, we retrieve for the mass and radius, the PT profile, the surface pressure, the molecular abundances and the cloud parameters. By covering a range of different wavelength ranges $(3-20 \mu \mathrm{m}, 6-17 \mu \mathrm{m})$, spectral resolutions $(R=20$, $35,50,100)$ and signal to noise ratios $(S / N=5,10,15,20)$, we obtain estimates for the technical requirements LIFE needs to meet to:

- Differentiate between a Venus- and an Earth-twin.

- Find evidence for clouds in Venus' atmosphere.

- Accurately characterize structure and composition of Venus' atmosphere.

This approach will show whether the previously determined technical requirements for LIFE (Konrad et al., in prep.) are sufficient. Many similar MIR retrieval studies have been performed for Earth-like exoplanets. However, to the best of our knowledge, no MIR retrieval studies exist for a Venus-like planet.

\section{Results}

In Figures 1 and 2, we provide first preliminary results, as a proof of concept from testing our approach for retrieving cloudy MIR spectra with our retrieval framework. We used a Venus-twin spectrum (range $=3-20 \mu \mathrm{m}, \mathrm{R}=50$, SNR $=20$ ) assuming a distance of $10 \mathrm{pc}$ from the Sun as input for this retrieval run. Please note, that for these retrieval validation runs, we only consider the planet's photon noise and not yet additional noise sources. 


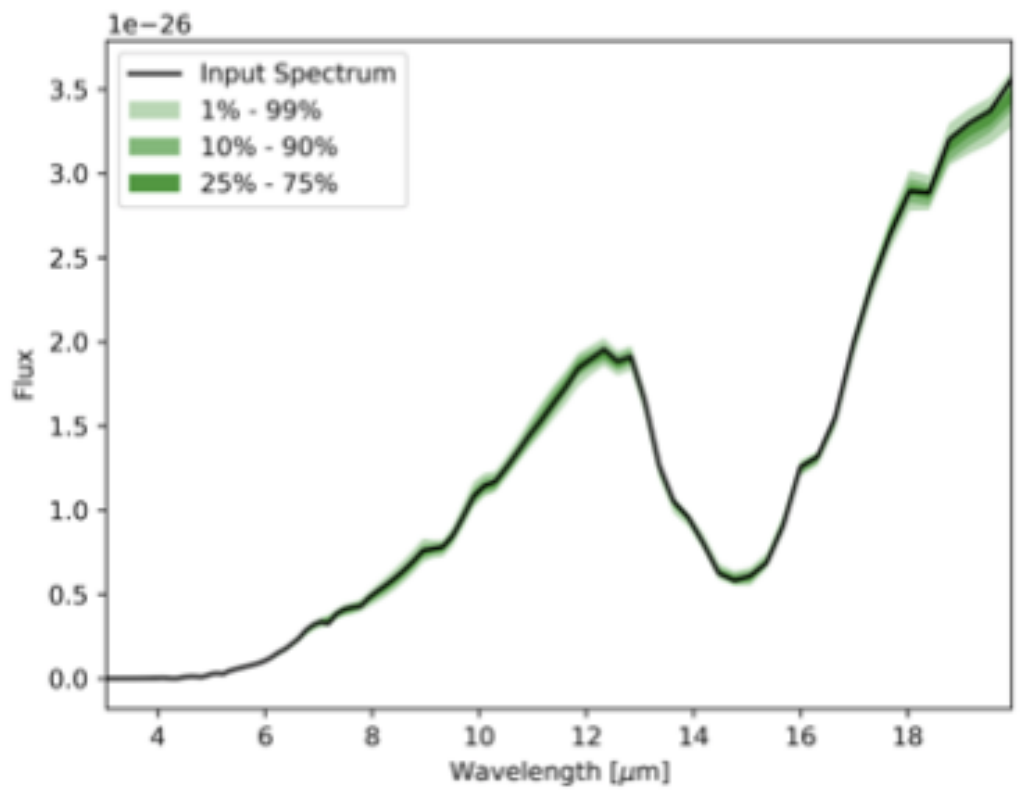

Figure 1: Uncertainty on the retrieval-fitted Venus spectra for a Venus-twin input spectrum covering $3-20 \mu \mathrm{m}, \mathrm{R}=50$ and an $\mathrm{S} / \mathrm{N}$ $=20$. The green shaded region marks the uncertainty on the fitted MIR spectrum. The black line represents the input spectrum.

Figure 1 shows the spectrum corresponding to the retrieved parameters in relation to the input spectrum. From this plot we see that our retrieval provides a good fit to the input spectrum. Figure 2 shows that the PT profile parameters are well retrieved for the upper atmosphere of Venus. Due to the opaque cloud layer in Venus' atmosphere at approx. $0.1 \mathrm{bar}$, the surface temperature and surface pressure are no longer retrievable (as can be seen from the cut in the green uncertainty at $0.1 \mathrm{bar}$ ). Instead we interpret the retrieved "surface" pressure as the pressure at which Venus' cloudy atmosphere becomes opaque. 


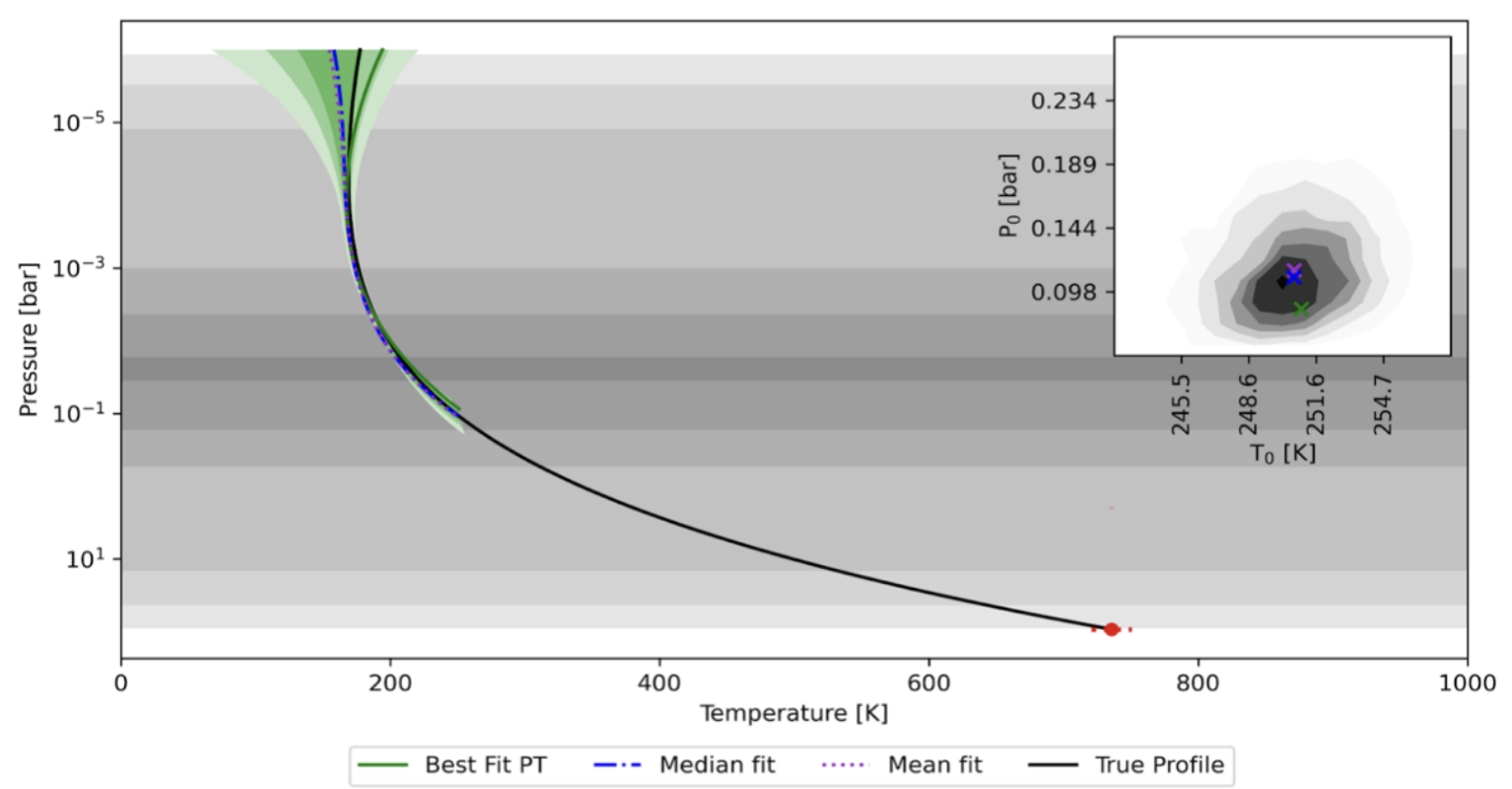

Figure 2: PT profile retrieved from a Venus-twin spectrum covering $3-20 \mu \mathrm{m}, \mathrm{R}=50$ and an $\mathrm{S} / \mathrm{N}=20$. The green shaded region marks the uncertainties on the PT profile fit. The gray shaded region represents cloud probability (dark $=$ high probability). The inlay plot shows the retrieved pressure and temperature at which the atmosphere becomes opaque.

With these first retrieval runs we demonstrate that our retrieval suite produces reliable results for cloudy atmospheres. Further studies are currently carried out and will be presented at the conference. Furthermore, we will address the points listed above and discuss how results from cloudy retrievals could be safely interpreted.

\section{References}

Quanz, S. P., et al., 2018, Proc. SPIE, 107011I

Bryson, S. et al., 2021, AJ, 161, 36. doi:10.3847/1538-3881/abc418

Quanz, S. P. et al., 2019, arXiv e-prints arXiv:1908.01316

Konrad, B. S., et al., 2020, European Planetary Science Congress

Mollière, P., et al., 2019, A\&A, 627:A67

Feroz, F., et al., 2009, MNRAS, 398(4):1601-1614

\section{Websites}

[a] https://www.luvoirtelescope.org

[b] https://www.jpl.nasa.gov/habex/ 
LIFE collaboration: https://www.life-space-mission.com/ 